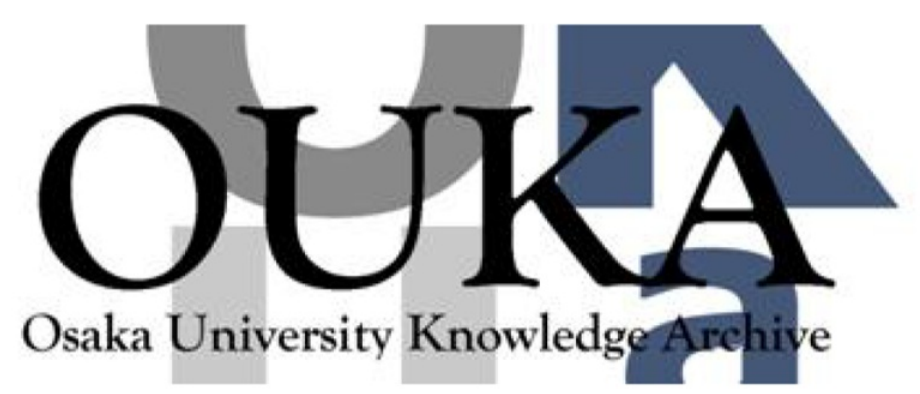

\begin{tabular}{|c|c|}
\hline Title & $\begin{array}{l}\text { Noncontact measurement of ultrasonic } \\
\text { attenuation during rotating fatigue test of } \\
\text { steel }\end{array}$ \\
\hline Author (s) & $\begin{array}{l}\text { Ogi, Horotsugu; Hirao, Masahiko; Minoura, } \\
\text { Kiyoshi }\end{array}$ \\
\hline Citation & Journal of Applied Physics. 81(8) p.3677-p.3684 \\
\hline Issue Date & 1997-04-15 \\
\hline oaire:version & VoR \\
\hline URL & https://hdl. handle. net/11094/84230 \\
\hline rights & $\begin{array}{l}\text { This article may be downloaded for personal use } \\
\text { only. Any other use requires prior permission } \\
\text { of the author and AIP Publishing. This article } \\
\text { appeared in Journal of Applied Physics, } 81(8) \text {, } \\
3677-3684 \text { (1997) and may be found at } \\
\text { https://doi.org/10.1063/1.365489. }\end{array}$ \\
\hline Note & \\
\hline
\end{tabular}

Osaka University Knowledge Archive : OUKA

https://ir. Library. osaka-u. ac. jp/

Osaka University 


\title{
Noncontact measurement of ultrasonic attenuation during rotating fatigue test of steel
}

\author{
Hirotsugu Ogi, ${ }^{a}$ Masahiko Hirao, and Kiyoshi Minoura \\ Faculty of Engineering Science, Osaka University, Machikaneyama 1-3, Toyonaka, Osaka 560, Japan
}

(Received 9 September 1996; accepted for publication 11 January 1997)

\begin{abstract}
Acoustic resonance technique has been applied to monitor the fatigue damage process of steel pipes exposed to rotating bending fatigue. The technique incorporates a superheterodyne spectrometer and an electromagnetic acoustic transducer (EMAT). The EMAT was newly developed for this purpose, and uses the magnetostrictive mechanism of ferromagnetic metals and excites and detects axial shear waves traveling around the sample pipe with axial polarization. Noncontact ultrasonic spectroscopy permits the accurate determination of the resonant frequency and the attenuation coefficient throughout the fatigue life. The attenuation coefficient shows a sharp peak around $80 \%-90 \%$ of the life. The evolution is interpreted as reflecting dislocation multiplication, depinning, and formation of cell structures, which is supported by transmission electron microscopy observations. () 1997 American Institute of Physics. [S0021-8979(97)04308-4]
\end{abstract}

\section{INTRODUCTION}

Nondestructive sensing of fatigue damage in metal structures has been an important subject for ensuring their safety and reliability. The goal is the detection of some indicative phenomena appearing much earlier than when the crack becomes visible with a conventional inspection technique, leading to the continuous monitoring of the damage progress. Ultrasonic methods can provide unique approaches for sensing the inside of solids. This study is devoted to the ultrasonic monitoring of the fatigue damage accumulation process during the rotating bending test of steel pipes. For this, we developed a transduction technique, relying of the magnetostrictive effect, to make use of the axial-shear acoustic resonance around the outer surface of a sample steel pipe. The fatigue monitoring is made possible by observing the resonant frequency and the attenuation measured with the method of electromagnetic acoustic resonance (EMAR). EMAR is a combination of the ultrasonic resonance and a noncontacting electromagnetic acoustic transducer (EMAT). The heavily and coherently overlapping echoes at a resonant state improves the signal-to-noise ratio to a large extent and compensates enough for the weak coupling efficiency with the EMAT. The noncontacting aspect has a large advantage for the attenuation measurement, because it eliminates energy losses associated with contact measurements. Otherwise, large energy losses occur at the sample-couplanttransducer interfaces. They generally exceed the damping loss within the sample.

The EMAR method was first proposed by Filimonov et al. ${ }^{1}$ and Nikiforenko et al. ${ }^{2}$ in 1971 . They detected the shear wave resonant spectra with an aluminum plate and obtained the anisotropic shear wave velocities. Twenty years later, Kawashima ${ }^{3}$ applied it to evaluate the rolling texture and the formability ( $r$-value) of thin steel sheets. Since then, this technique has developed to accommodate various shapes of objects including spheres, circular rods and pipes, hexagonal rods, etc. Johnson et al. measured elastic constants of metal spheres ${ }^{4}$ and then evaluated the hardening depth of steel rods. ${ }^{5}$ The accuracy has been considerably raised and

${ }^{a)}$ Electronic mail: ogi@me.es.osaka-u.ac.jp the EMAR method is applicable even to acoustoelastic stress measurements, in which we need to determine ultrasonic velocities to the fractional order of accuracy of $10^{-6}$. Hirao et al. measured the two-dimensional stress profile in thin metal plates ${ }^{6}$ and the axial stresses in railroad rails. ${ }^{7}$ Ogi et al. expanded the technique to the absolute measurement of the ultrasonic attenuation ${ }^{8}$ and determined the grain size of carbon steels from the frequency dependence of the attenuation. ${ }^{9}$

The axial shear wave propagates along the circumference of a cylindrical rod or pipe with the polarization in the axial direction. This mode of the elastic wave is not excited with conventional transduction methods. The major advantage is the capability of evaluating the inhomogeneous microstructure in the radial direction by using the different resonant modes. Each resonant mode shows the amplitude peak at a specific radius, to which the measurement result is selectively sensitive. Johnson et al. ${ }^{5}$ first detected the EMAR spectra of the axial shear resonance from a cylindrical rod. They employed the Lorentz force mechanism to design the EMAT, which was built with many permanent magnets periodically spaced around the rod and a solenoidal coil surrounding the rod surface. The spacing of the magnets corresponds to the wavelength of the shear horizontal (SH) wave to be generated. When a higher spatial resolution in the radial direction is required, the EMAT designing involves using a larger number of thinner permanent magnets. Such an array of thin magnets cannot introduce a strong field in the sample surface and is vulnerable to liftoff (air gap). In the present study, we invented an alternative EMAT relying on the magnetostrictive effect in ferromagnetic metals. This EMAT consists of a solenoidal coil to apply a static field along the axial direction of a sample pipe and a meanderline coil to induce the dynamic field perpendicular to the static field. The meanderline spacing determines the wavelength of the shear wave and then determines the spatial resolution of the inspection. Recent printed circuit techniques make it easy to fabricate the meanderline coil with smaller spacings for the evaluation of the sample material with higher radial resolution. 


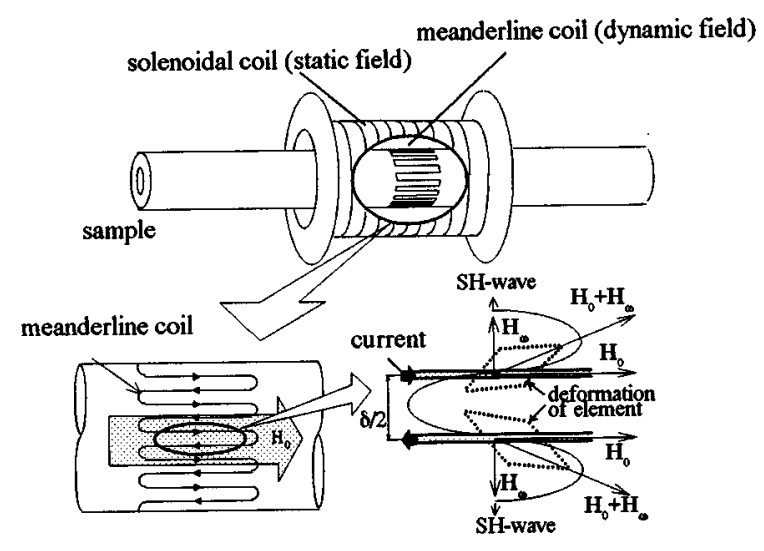

FIG. 1. Principle of the EMAT for axial shear resonance. $\mathbf{H}_{0}$ is the static field applied by the solenoidal coil, and $\mathbf{H}_{\omega}$ is the dynamic field caused by the meanderline coil.

The influence of metal fatigue on the ultrasonic properties is a long-running problem in the field of physical acoustics. ${ }^{10-17}$ Among others, the ultrasonic attenuation (or internal friction) has been used to identify the fatigue stage of metals. Previous work ${ }^{10-14}$ showed that, in high cycle fatigue testing, the attenuation rate increases slightly at the beginning of the fatigue life and, after experiencing a long stable period, it remarkably increases just prior to failure. The initial increase is explained by the increase of the dislocation and the final increase is attributed to the occurrence of microcracks and their subsequent growth.

The present axial-shear EMAR measurement reveals a novel feature of the ultrasonic-fatigue relation. A sharp attenuation peak and a velocity depression always appear at $80 \%-90 \%$ of the life; this could be a warning to approaching fracture. With the help of optical micrographs, transmission electron micrographs, and replica films, we conclude that the change of the ultrasonic properties is caused mainly by the change of the dislocation structure. The acoustic data allow the calculation of the dislocation density and the loop length on the basis of the Granato-Lücke string model. ${ }^{18}$

\section{MEASUREMENT OF AXIAL SHEAR RESONANCE}

\section{A. Magnetostrictively coupled EMAT}

When a magnetic field is applied to a ferromagnetic material, the dimensional change (strain) arises in the field direction; this is called magnetostriction. The magnitude of static magnetostriction is on the order of $10^{-6}$ in common steels. When the applied field oscillates at a high frequency, so does the magnetostriction, giving rise to the ultrasonic signal.

Figure 1 illustrates the magnetostrictively coupled EMAT. It consists of the solenoidal coil to introduce the static field in the axial direction of a steel pipe (or rod), and the meanderline coil of equal spacings surrounding the sample surface. Applying a sinusoidal current to the meanderline coil, it produces the dynamic field in the circumferential direction. The dynamic field is superimposed on the static field and the total field acts along the vector sum of these two fields. The magnetostriction takes place in the di-

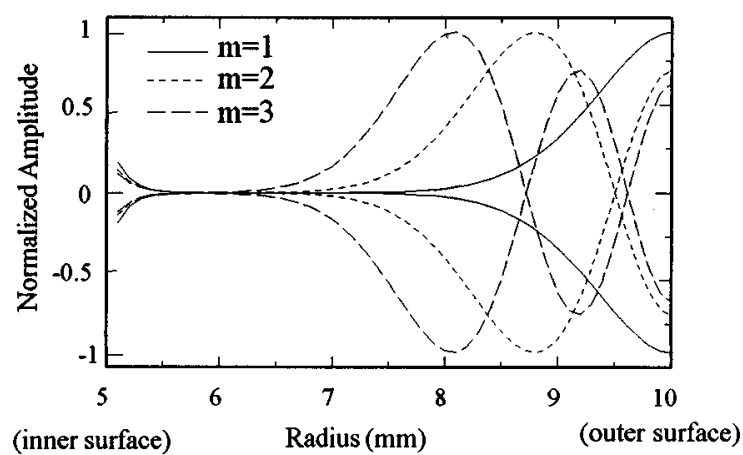

FIG. 2. Amplitude distributions at three resonant modes for the steel pipe of $20 \mathrm{~mm}$ outer diameter and $10 \mathrm{~mm}$ inner diameter $(n=48)$.

rection of the total field, depending on its magnitude. Then, the surface element undergoes an oscillating shear deformation. The SH wave is, thus, excited and propagated along the circumference having the axial polarization. ${ }^{19,20}$ The same meanderline coil receives the wave in the reversed process, although the receiving mechanism is not well understood at present. The EMAT has the optimum excitation efficiency when the wavelength is tuned to the meanderline spacing.

\section{B. Axial shear resonance spectrum}

Resonant modes of the axial shear wave can be calculated by solving the elastodynamic equation with the boundary conditions for the free surfaces. For a pipe, we have the frequency equation (resonant condition) as

$$
\begin{aligned}
& \left\{\frac{n J_{n}\left(k R_{a}\right)}{k R_{a}}-J_{n+1}\left(k R_{a}\right)\right\} \cdot\left\{\frac{n Y_{n}\left(k R_{b}\right)}{k R_{b}}-Y_{n+1}\left(k R_{b}\right)\right\} \\
& -\left\{\frac{n J_{n}\left(k R_{b}\right)}{k R_{b}}-J_{n+1}\left(k R_{b}\right)\right\} \cdot\left\{\frac{n Y_{n}\left(k R_{a}\right)}{k R_{a}}\right. \\
& \left.-Y_{n+1}\left(k R_{a}\right)\right\}=0,
\end{aligned}
$$

where $J_{n}$ and $Y_{n}$ are the $n$th Bessel function of the first and the second kinds, respectively; $R_{a}$ and $R_{b}$ are the outer and inner radii $\left(R_{a}>R_{b}\right)$, and $k$ is the wave number $(k=\omega / c)$, where $\omega$ is the angular frequency and $c$ is the shear wave velocity. Integer $n$ is determined by

$$
n \sim 2 \pi R_{a} / \delta
$$

where $\delta$ is the meanderline period (Fig. 1). Numerical solutions to Eq. (1) provide a series of resonant frequencies, $f_{m}^{(n)}$, with another integer $m$ identifying the overtones. Figure 2 shows the calculated displacement amplitude for the first three resonant modes at $n=48$ (i.e., $f_{1}^{(48)}, f_{2}^{(48)}$, and $f_{3}^{(48)}$ ) for a steel pipe with $R_{a}=10 \mathrm{~mm}$ and $R_{b}=5 \mathrm{~mm}$. The oscillation of the first mode is restricted to the outer surface region and, as the mode becomes a higher overtone, the peak amplitude moves to the inside of the pipe. It is then possible to evaluate the microstructure gradient in the radial direction using the different resonant modes. The integer $n$ determines 


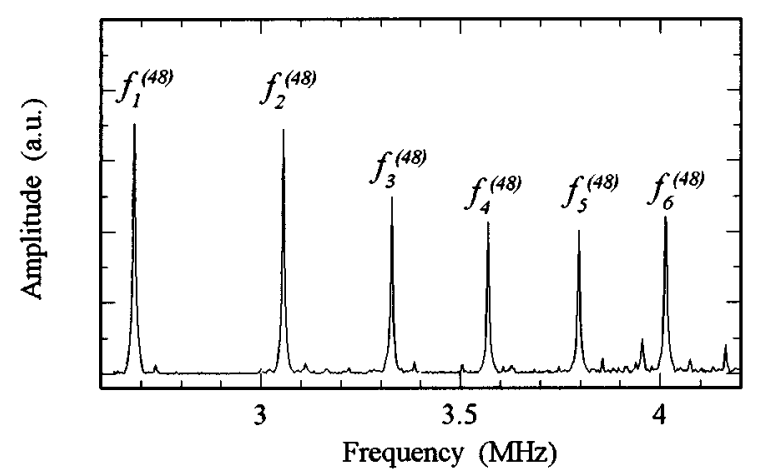

FIG. 3. Measured resonant spectrum of the steel pipe.

the penetration depth of the fundamental mode and, therefore, governs the spatial resolution of the measurement. The fundamental mode $(m=1)$ with larger $n$ is suitable for the characterization focused on the most outer region, where the damage develops preferentially in the rotating fatigue due to the higher bending stress and a reduced constraint on deformation.

\section{Resonant frequency and attenuation coefficient}

We measured the resonant frequency and the attenuation coefficient using a superheterodyne spectrometer. ${ }^{21}$ The measurement proceeds in several steps, but the procedures are programmed in a single run, which finishes in a short time ( $\sim 10 \mathrm{~s})$. First, we drive the meanderline coil of $20 \mathrm{~mm}$ width with high-power and long rf bursts. The duration is set to $100 \mu \mathrm{s}$, being about five times of the round-trip time for the surface SH wave around the pipe. Just after the excitation, the same coil detects the highly overlapping shear wave signals. The received signals are sent to the quadrature phase sensitive detectors, and then the amplitude and phase spectra are extracted from the signal within an integrator gate. ${ }^{6} \mathrm{We}$ obtain a resonant spectrum by sweeping the frequency of the input rf burst and determining the amplitude as a function of the operating frequency. Figure 3 shows a typical axial shear resonant spectrum measured for a steel pipe of $R_{a}=10 \mathrm{~mm}$ and $R_{b}=5 \mathrm{~mm}$, where we used the magnetostrictively coupled EMAT of $n=48$. We used the meanderline coil of $20 \mathrm{~mm}$ width printed on a polyimide sheet. We determine the resonant frequency by fitting the Lorentzian function to the measured spectra around each peak and calculated the center axis. The resonant frequencies are observed in uneven intervals (cf. Refs. 6-9). The measured resonant frequencies agree with the calculation based on frequency Eq. (1) within the error band of $1 \mathrm{kHz}$.

We then measure the ringdown curve by driving the same EMAT with the measured resonant frequency and stepping the narrow gate along the time axis. ${ }^{9}$ Figure 4 shows the measured ringdown curve for the fundamental mode in Fig. 3 . We finally obtain the time coefficient by fitting an exponential decay to the measured ringdown curve and calculating the time coefficient. The as-measured time coefficient contains the attenuation in the sample and also the diffraction loss, which occurs due to the beam spreading in the axial

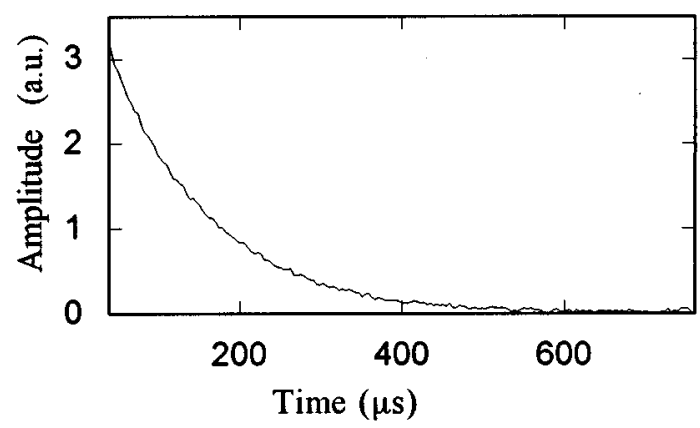

FIG. 4. Measured ringdown curve at the fundamental mode $\left(f_{1}^{(48)}\right)$.

direction. The diffraction effect is, however, not significant, because this is a one-dimensional diffraction from the wide meanderline coil. Furthermore, we are mainly interested in the relative change of the attenuation throughout the fatigue life.

\section{MONITORING OF ROTATING FATIGUE DAMAGE}

\section{A. Setup of four-point bending fatigue test}

Figure 5 is a sketch of the rotating bending setup. Tested were extruded carbon steel pipes $1 \mathrm{~m}$ long, $20 \mathrm{~mm}$ outer diameter, and $10 \mathrm{~mm}$ inner diameter. The carbon content is 0.23 mass \%. The motor rotates the pipe at $240 \mathrm{rpm}(4 \mathrm{~Hz})$. The four-point bending stress is applied by external weights. The maximum bending stress is $240 \mathrm{MPa}$ on the outer surface, which is about $80 \%$ of the yield stress. We introduced a smooth shallow notch of $30 \mu \mathrm{m}$ deep and $1 \mathrm{~mm}$ wide around the circumference at the center of the pipe. The surrounding area was electrolytically polished in a solution of 9 parts of $\mathrm{CH}_{3} \mathrm{COOH}$ and 1 part $\mathrm{HClO}_{4}$ to produce a surface of high quality.

\section{B. Evolution of the resonant frequency and the attenuation coefficient}

The resonant frequencies and the attenuation coefficients at the lowest three modes were measured during the fatigue test. The static field from the solenoidal coil was fixed to $0.18 \mathrm{kOe}$. At each measurement, we interrupted the rotation and bending to make a brief measurement and then restarted fatiguing. We repeated this procedure until failure occurred.

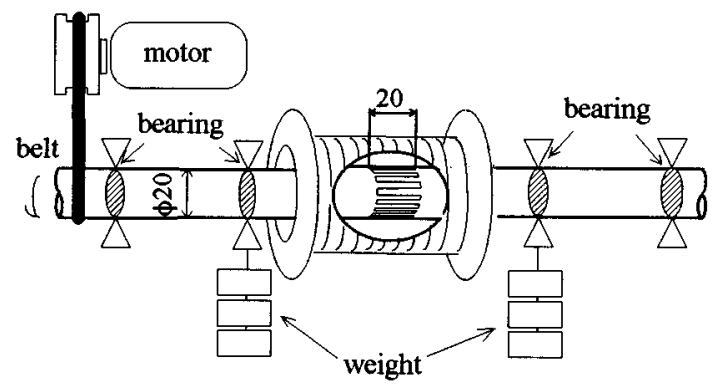

FIG. 5. Setup of rotating bending fatigue test. 


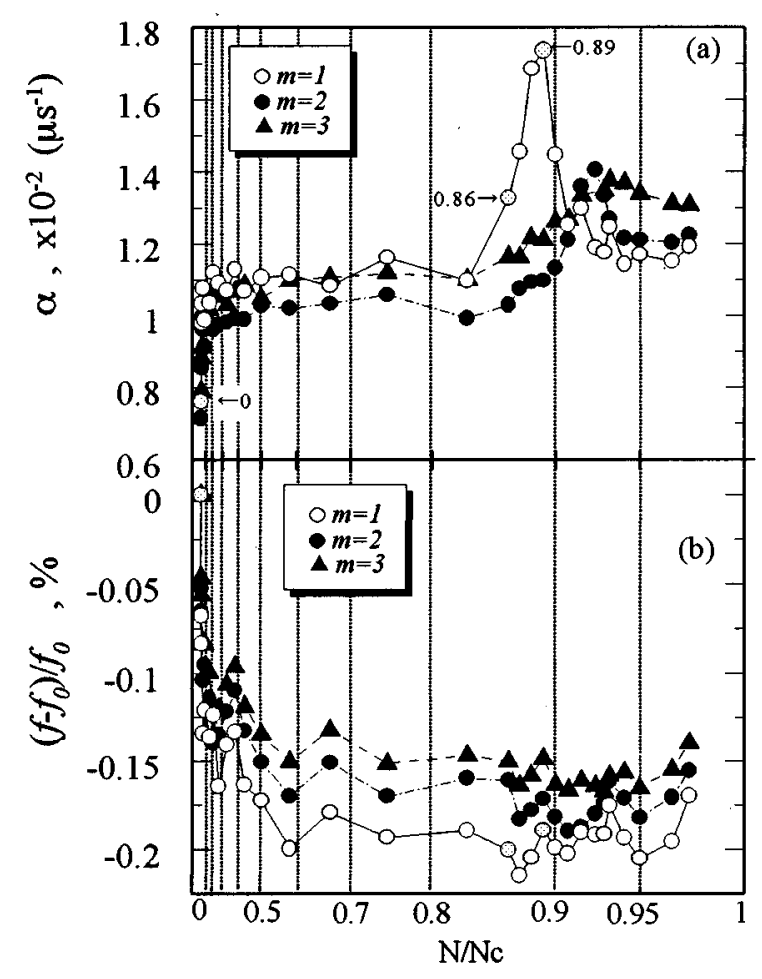

FIG. 6. Evolution of attenuation coefficient and resonant frequency shift during the fatigue life ( $N_{c}=610000$ cycles). $f_{0}$ is the original resonant frequency.

Figure 6 is the typical evolution of the attenuation coefficient and the resonant frequency shift. The horizontal axis is exponentially elongated as the cycle number becomes larger, because the important behaviors of the ultrasonic properties are observed near the end of the fatigue, which would not be clearly visible with the linear scale of the cycle number. The resonant spectra and the ringdown curve for the fundamental mode at three representative fatigue stages are given in Fig. 7. We carried out similar tests for 13 samples and observed the same features of ultrasonic-fatigue interaction, although the total numbers of cycles to fracture varied between 50000 and 650000 cycles, probably depending on the surface condition and the microstructure. General observations are:

(i) At the initial stage, the attenuation coefficient increases and the resonant frequency decreases for all three resonant modes.

(ii) Both the attenuation and the resonant frequency change little for nearly half the fatigue life.

(iii) Around $80 \%-90 \%$ of the life, the attenuation coefficient jumps to a maximum value and then it levels off immediately. Simultaneously, the resonant frequency experiences a small depression and then becomes stable.

(iv) The attenuation coefficient changes more noticeably than the resonant frequency. The attenuation peak appears to be more than twice as high as the original attenuation, while the variation in the resonant frequency is limited to within $0.2 \%-0.3 \%$.

(v) The attenuation peak of the fundamental mode is observed to be of the largest magnitude and earliest among three modes, as has been expected.

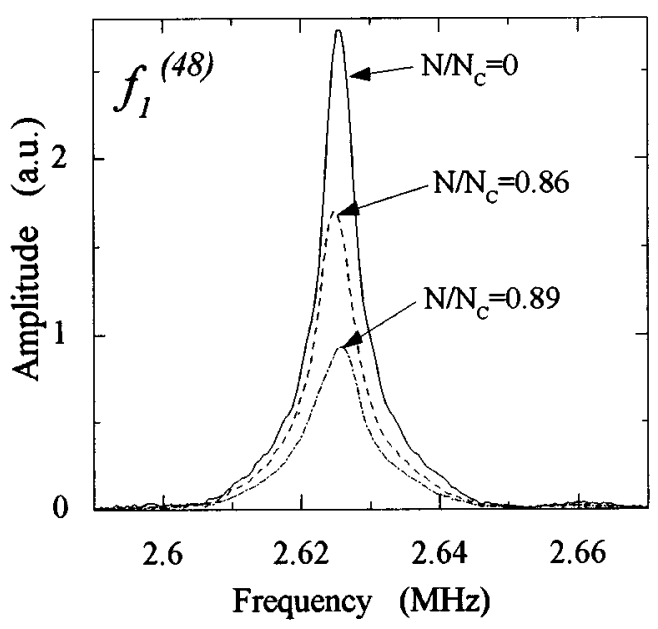

(a)resonant spectrum

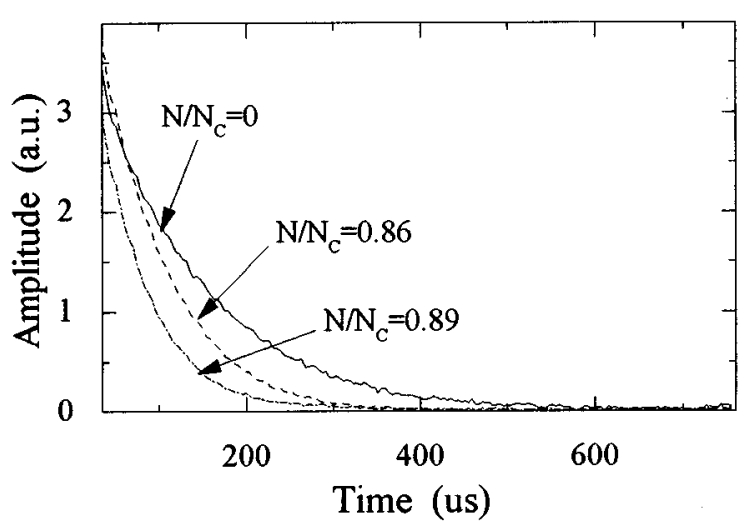

(b)rindown curve

FIG. 7. (a) Resonant spectra and (b) ringdown curves at three fatigue cycles of Fig. 6.

Despite extensive research on the relation between the attenuation and fatigue damage, there has been no report of this attenuation peak. This is partly because the peak is so sharp and is likely to be overlooked by the conventional techniques. The second reason is the high sensitivity of the method. Owing to the noncontact transduction, the EMAR method is capable of providing the absolute value of the attenuation coefficient, which represents the damping character in the material. The EMAR excludes extra energy losses, which are indispensable when using the contact transducers and would very possibly mask the minute indications of the microstructural changes. Furthermore, the contactless transducer yields highly reproducible data independent of the measuring conditions. Unfavorable factors like liftoff and surface roughness will diminish the spectrum intensity and the amplitude of the ringdown curve, but they will not change the decay rate, from which the attenuation coefficient is determined. Equally important is the concentration of the ultrasonic deformation to the outer surface of the sample pipe. This is made possible by incorporating the magneto- 


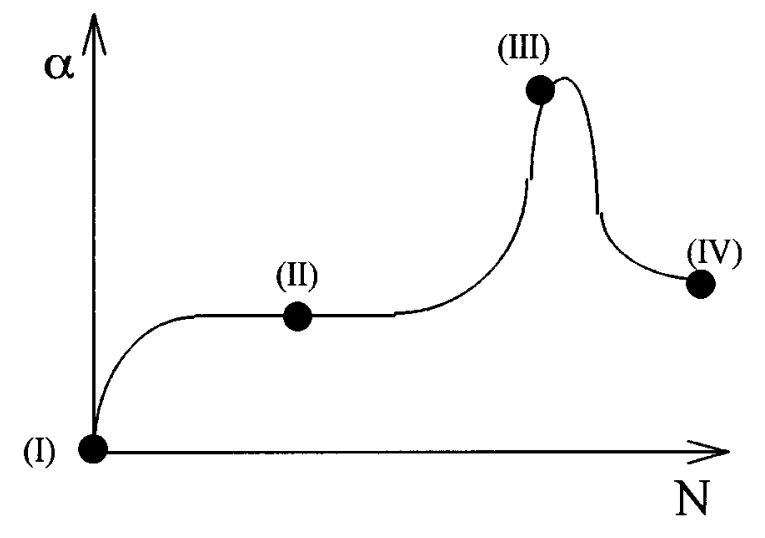

FIG. 8. Four fatigue stages based on the attenuation character.

strictive mechanism for the transduction and using the fundamental axial shear mode at large $n$.

\section{MICROSTRUCTURAL CHANGES}

Seeking a satisfactory explanation to the above behavior, we investigated the microstructure and its change during the fatigue test. We focused on four fatigue stages; stage I: before the test, stage II: in the stable region, stage III: just before the fundamental mode shows the attenuation peak, and stage IV: after the attenuation peak of the fundamental mode (Fig. 8). The optical micrograph serves to observe the cracks and the grain size and the transmission electron micrograph (TEM) to know the dislocation structure. For these observations, we truncated the fatigue loading and extracted specimens in the vicinity of the notch. The replica method was also used to trace the surface crack growth throughout the test.

\section{A. Optical micrography observation}

Rectangular specimens including such from the notched area of $3 \times 3 \times 5 \mathrm{~mm}^{3}$ were sampled and examined over the cross-sectional area of $3 \times 5 \mathrm{~mm}^{2}$ normal to the circumferential direction. The specimens were etched in a solution of 95 parts of $\mathrm{C}_{2} \mathrm{H}_{5} \mathrm{OH}$ and 5 parts of $\mathrm{HNO}_{3}$.

We found the appearance of small cracks along the notch bottom as early as $20 \%-30 \%$ of the total life. Besides this, we saw no significant difference among stages I-III. However, a grain refinement was seen in the surface region at stage IV. The grain size was $12-14 \mu \mathrm{m}$ through stages I-III, and also at the interior region of stage IV. But, it decreases to around $9 \mu \mathrm{m}$ in the proximity of the notch bottom at stage IV. This can be explained by the developing subgrain structure due to the cyclic loading. ${ }^{10}$

\section{B. TEM observation}

We made three slices of $0.2 \mathrm{~mm}$ thickness from each specimen using the electrical discharge wire and mechanically polished them to $0.1 \mathrm{~mm}$ thickness. The slice faces were oriented parallel to the original sample surfaces. They were then given an electrolytic polishing in a solution of 9 parts of $\mathrm{CH}_{3} \mathrm{COOH}$ and 1 part of $\mathrm{HClO}_{4}$ to make small center holes, around which the TEM images were obtained.
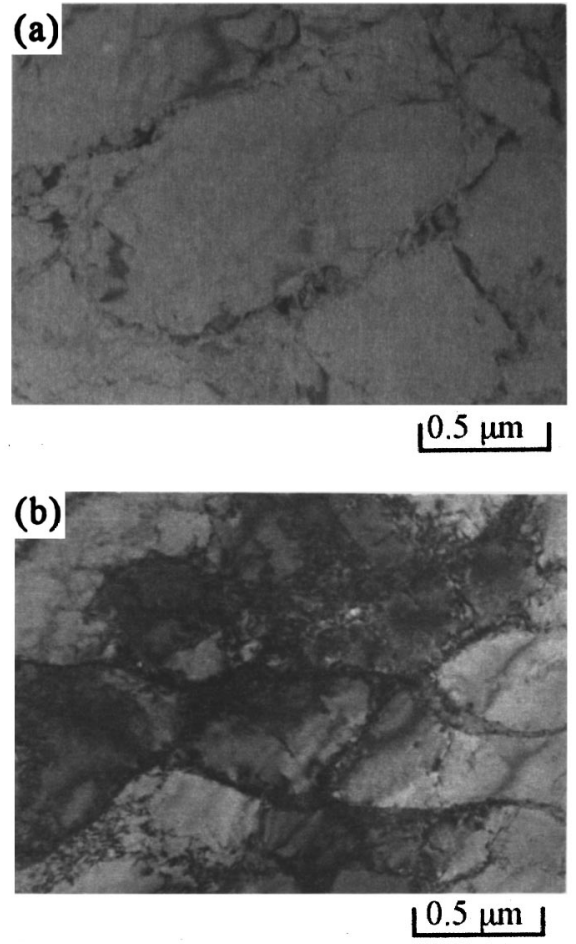

FIG. 9. TEM images of the notch bottom from (a) stage I and (b) stage III.

Figure 9 shows representative micrographs for stages I and III. Examining a large number of such TEM images, we revealed that the dislocation density continued increasing during the stable period (stage II). At stage III, the dislocations had multiplied to a considerable level, developing the cell structures, which were absent at stages I and II. Cell structures developed to a larger extent at the surface rather than in the interior. At stage IV, the dislocation density increased to the maximum and occupied the whole viewing area.

\section{Replication}

The replication technique provided the in situ observation of the macroscopic cracking at the notch bottom. We used acetyl cellulose film and methyl acetate for this purpose. To take the replicas, we applied the bending stress to open the cracks. Figure 10 compares the EMAR measurements for the fundamental mode with the maximum crack length obtainable from the replica films.

\section{DISCUSSION}

We consider dislocation damping, scattering at grain boundaries, and fatigue cracks as the potential sources contributing to the evolution of the ultrasonic properties ( $\alpha$ and $f$ ) during the fatigue tests.

The classical string model ${ }^{18}$ states that in the lower frequency region, the dislocation serves to change the attenuation coefficient $\alpha_{\text {dis }}$ and the velocity $v$ via 


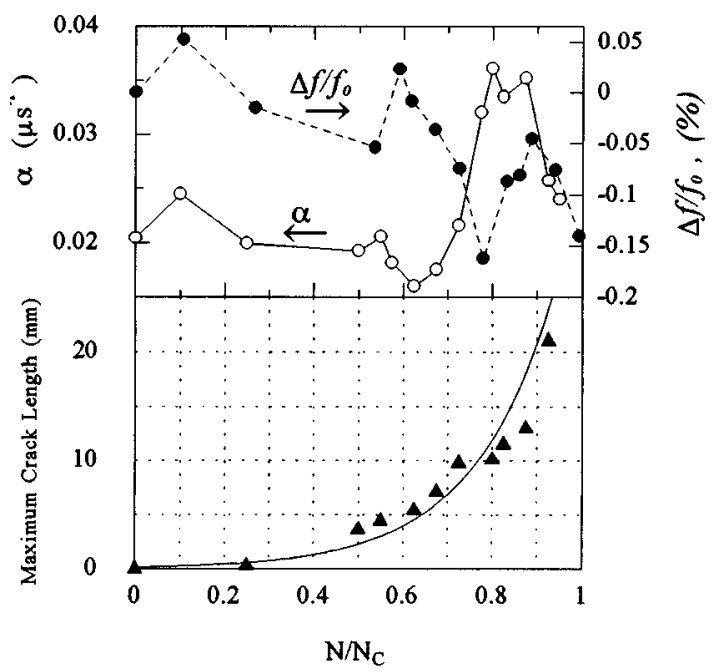

FIG. 10. Relation between the attenuation change and velocity shift of the fundamental mode, and the maximum crack length on the notch bottom $\left(N_{c}=210000\right.$ cycles $)$.

$$
\begin{aligned}
& \alpha_{\mathrm{dis}}=\left(\frac{16 G B b^{2}}{\pi^{4} C^{2}}\right) \Lambda L^{4} f^{2}, \\
& \frac{v_{0}-v}{v_{0}}=\left(\frac{4 G b^{2}}{\pi^{4} C}\right) \Lambda L^{2},
\end{aligned}
$$

where $G$ is the shear modulus, $B$ the specific damping constant, $\Lambda$ the dislocation density, $L$ the dislocation loop length, and $b$ the Burgers vector. $C$ denotes the line tension of an edge dislocation and is expressed by $2 G b^{2} / \pi(1-\nu)$ with Poisson's ratio $\nu{ }^{22} v_{0}$ is the dislocation-independent velocity of the material, which in principle would have to be measured at such a high frequency that the dislocation cannot oscillate responding to the elastic waves.

Grain boundaries can also be the source of ultrasonic attenuation. An ultrasonic wave propagating in a polycrystalline metal undergoes scattering due to the different crystallographic orientations of adjacent grains, causing an energy loss. When the probing wavelength is much larger than the grain size (Rayleigh scattering regime), the attenuation coefficient $\alpha_{s}$ is expressed by ${ }^{23}$

$$
\alpha_{s}=S D^{3} f^{4},
$$

where $S$ is the scattering factor and $D$ the average grain size. The grain size makes a contribution to the attenuation through $D^{3}$. The effect is, however, not the principal mechanism in the present case. Based on a grain size of about $9 \mu \mathrm{m}$ near the surface in stage IV and $14 \mu \mathrm{m}$ in stage III, and using the scattering factor $S=2.25 \times 10^{-10} \mu \mathrm{s}^{3} / \mu \mathrm{m}^{3}$ (Ref. 9) for the shear wave in steels at a frequency of $2.7 \mathrm{MHz}$ for the fundamental mode, we calculate $\alpha_{s}=3.3 \times 10^{-5} \mu \mathrm{s}^{-1}$ in stage III and $\alpha_{s}=8.7 \times 10^{-6} \mu \mathrm{s}^{-1}$ in stage IV. These values are negligible compared with the observed attenuation of the order of $10^{-3}-10^{-2} \mu \mathrm{s}^{-1}$.

Cracks also cause attenuation through scattering. But, the effect is thought to be negligible, too. The cracking is a small event, considering that the axial shear wave is probing

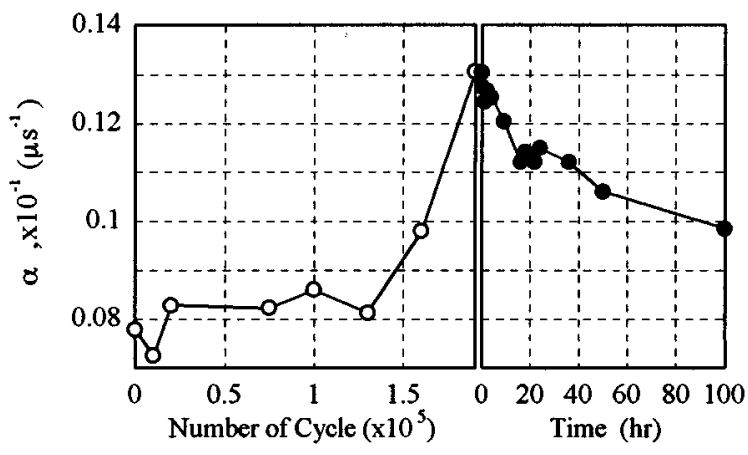

FIG. 11. Measurement of the attenuation recovery for the fundamental mode.

a large cylindrical area of $20 \mathrm{~mm}$ width and also that the propagation direction is parallel to the cracks. Figure 10 compares the evolutions of $\alpha$ and $f$ of the fundamental mode with the maximum crack length along the notch bottom. While the crack length monotonously increases with the cycle number, $\alpha$ shows a complicated change including the peak. We observe no correlation between the attenuation coefficient and the maximum crack length, supporting that the crack is not the main factor in changing attenuation.

To investigate the attenuation phenomena in more detail, we carried out a supplemental experiment. When the fundamental mode exhibited the peak attenuation, we stopped rotating the sample pipe. We then kept it in the stress-free condition and monitored the temporal change of attenuation at room temperature. In Fig. 11, approaching the peak, the increase in $\alpha$ amounts to $0.05 \mu^{-1}$ relative to the stable period and approximately $65 \%$ of this increase is released in the subsequent $100 \mathrm{~h}$. It is then apparent that the crack is not the principal cause of the attenuation development. The above change of $\alpha$ can be explained by the dislocation recovery, ${ }^{24}$ which occurs accompanying the dislocation pinning by the impurities. The impurities migrate to the distorted regions around the dislocations and pin the dislocations, thereby shortening the loop length $L$ and diminishing the attenuation coefficient. We, furthermore, annealed the same sample at $220^{\circ} \mathrm{C}$ for $1 \mathrm{~h}$. The attenuation coefficient recovered to $0.07 \mu \mathrm{s}^{-1}$, being less than the original value before fatiguing.

From these observations, we conclude that the dislocation mobility is the principal source of the attenuation change and the velocity shift in the present fatigue tests. It is obvious that the crack growth governs the fatigue life. Dislocations play an important role in crack growth by producing the extremely high stress regions around the crack tip. ${ }^{10,25} \mathrm{On}$ the other hand, the evolutions of ultrasonic properties ( $\alpha$ and $f$ ) are explained by the dislocation mobility. The EMAR method measures the ultrasonic properties averaged over a volume along the sample surface. Because the attenuation peak occurs at approximately the same fraction of the cycles to failure, there should be an unknown link between the global dislocation formation and its local contribution to the crack extension in the fatigue process.

Assuming that the dislocation is the only responsible mechanism for the phenomena, we solved simultaneously 


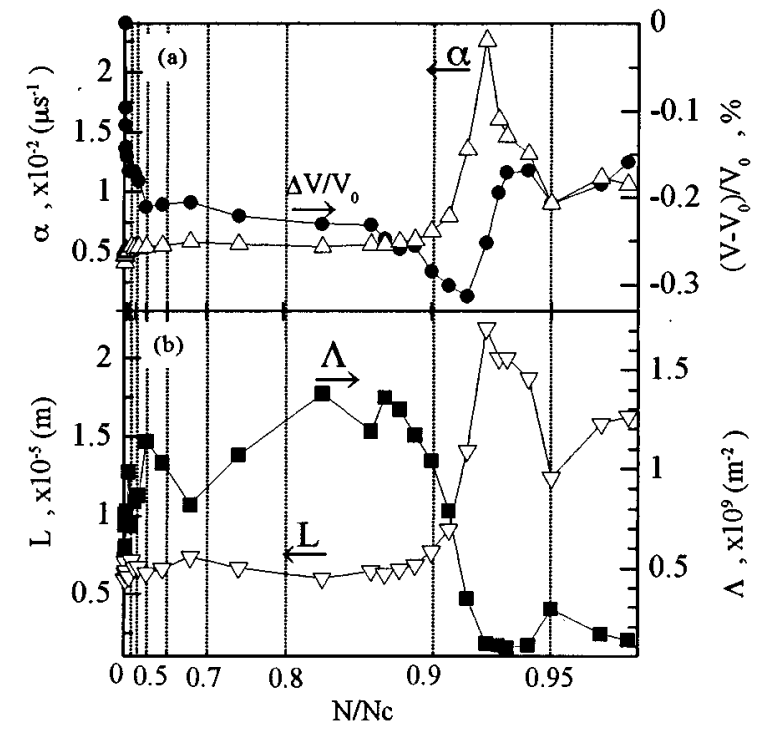

FIG. 12. (a) Measurement of the attenuation coefficient and velocity shift for the fundamental mode, and (b) the changes of the dislocation density $(\Lambda)$ and the dislocation loop length $(L)$ calculated from the measurement data.

Eq. (3) with the measured $\alpha$ and $\Delta v / v(=\Delta f / f)$ to determine the dislocation density $\Lambda$ and the loop length $L$ at each stage. For simplicity, we have neglected the dislocations that exist originally. The variations of the $\alpha$ and $\Delta v / v$ are, therefore, related to the changes of $\Lambda$ and $L$. The parameters used in the calculations are $G=80 \mathrm{GPa}, b=3.0 \times 10^{-10} \mathrm{~m}, \nu=0.3$, and $B=10^{-3} \mathrm{~N} \mathrm{~s} / \mathrm{m}^{2}$.

We observe in Fig. 12 that the order of magnitude of $\Lambda$ is too small, while that of $L$ is as expected from the TEM observations. Note that only a small fraction of dislocations participate in the interaction with the ultrasonics and contribute to modifying the attenuation and velocity. Up to $N / N_{c} \sim 0.85, \Lambda$ increases gradually and $L$ remains almost unchanged. During the stable period, the dislocations multiply steadily. At the same time, the impurities pin the dislocation lines and decrease the loop length $L$, which cancels the attenuation enhancement due to the dislocation multiplication. Approaching the attenuation peak, $\Lambda$ drops and $L$ rises abruptly. This manifests the depinning of dislocations, that is, the dislocations are released from the pinning points. Up to this stage, the dislocation has become highly dense and the back stress reaches the extreme high level. We interpret the attenuation peak as the indication of the ongoing depinning process, which is followed by the dislocation tangling process toward the cell structure. Knowing that the cell walls are less mobile, we recognize that the cell structure has fully developed at the end of the attenuation peak. The TEM observations support this explanation; detailed cell structures were visible at stage III, but they were hardly seen in stages I and II. After the peak, the dislocations spread and pile up against the obstacles, such as grain boundaries, precipitates, and mainly other dislocations. The piling up of dislocations limits the dislocation mobility and decreases the attenuation observed at stage IV.

\section{CONCLUSIONS}

The magnetostrictively coupled EMAT was developed to generate the axial-shear acoustic resonance in a steel pipe. The EMAT consists of the solenoidal coil and the meanderline coil, and is capable of detecting high-frequency axial shear waves $(\sim 2.7 \mathrm{MHz})$ with a low magnetic field $(\sim 0.18 \mathrm{kOe})$. Each resonant mode of the axial shear wave has a unique propagation region in the sample. The lowest mode is concentrated near the outer surface. The spatial resolution to the radius direction was less than $1 \mathrm{~mm}$ with the present EMAT. The meandering period needs to be further reduced to investigate the dislocation-ultrasonic interaction in more detail.

The attenuation coefficient and the resonant frequencies can be determined with an accuracy enough to monitor the rotating fatigue damage accumulation in the steel pipe. They showed characteristic changes corresponding to a number of fatigue stages. In particular, the attenuation coefficient showed a pronounced peak at $80 \%-90 \%$ of the fracture life. The evolution of ultrasonic properties was interpreted in terms of the dislocation mobility. The density and the average loop length for the effective dislocations were calculated from the acoustic data using the classical string model. They gave some insight into the dislocation formation, which is compatible with TEM observations. Future work will involve the comparison with other quantitative techniques that provide information of dislocation behavior.

\section{ACKNOWLEDGMENTS}

The authors are grateful to Professor H. Mori of the Research Center for Ultra-High Voltage Electron Microscopy, Osaka University, who kindly helped us obtain the TEM images in his facility.

${ }^{1}$ S. A. Filimonov, B. A. Budenkov, and N. A. Glukhov, Sov. J. Nondestr. Test. 1, 102 (1971).

${ }^{2}$ Z. G. Nikiforenko, N. A. Glukhov, and I. I. Averbukh, Sov. J. Nondestr. Test. 4, 427 (1971).

${ }^{3}$ K. Kawashima, J. Acoust. Soc. Am. 87, 681 (1990).

${ }^{4}$ W. L. Johnson, S. J. Norton, F. Bendec, and R. Pless, J. Acoust. Soc. Am. 91, 2637 (1992).

${ }^{5}$ W. L. Johnson, B. A. Auld, and G. A. Alers, Review of Progress in QNDE, edited by D. O. Thompson and D. E. Chimenti (Plenum, New York, 1994), Vol. 13, p. 1603.

${ }^{6}$ M. Hirao, H. Ogi, and H. Fukuoka, Rev. Sci. Instrum. 64, 3198 (1993).

${ }^{7}$ M. Hirao, H. Ogi, and H. Fukuoka, Res. Nondestr. Eval. 5, 211 (1994).

${ }^{8}$ H. Ogi, M. Hirao, T. Honda, and H. Fukuoka, Review of Progress in QNDE, edited by D. O. Thompson and D. E. Chimenti (Plenum, New York, 1995), Vol. 14, p. 1601.

${ }^{9}$ H. Ogi, M. Hirao, and T. Honda, J. Acoust. Soc. Am. 98, 458 (1995)

${ }^{10} \mathrm{~V}$. S. Ivanova, The Role of Dislocations in the Strengthening and Failure of Metals, National Lending Library for Science and Technology, 1967.

${ }^{11}$ F. A. Firestone, Nondestr. Test. 7, 5 (1948).

${ }^{12} \mathrm{R}$. Truell and A. Hikata, Fatigue and Ultrasonic Attenuation, ASTM-STP, 213 (1957).

${ }^{13}$ W. J. Bratina and D. Mills, Can. Metall. Q. 1, 83 (1962).

${ }^{14}$ Z. Pawlowski, Proc. Fourth Int. Conf. on Nondestr. Test. (Butterworths, London, 1964), p. 192.

${ }^{15}$ S. Golovin and A. Puskar, Mater. Sci. Forum 119/121, 407 (1993).

${ }^{16}$ G. Fei and Z. Zhu, Phys. Status Solidi A 140, 119 (1993).

${ }^{17}$ Z. Zhu and G. Fei, J. Alloys Compd. 211/212, 93 (1994).

${ }^{18}$ A. Granato and K. Lücke, J. Appl. Phys. 27, 583 (1956).

${ }^{19}$ R. B. Thompson, Appl. Phys. Lett. 34, 175 (1979). 
${ }^{20}$ H. Ogi, K. Minoura, and M. Hirao, Review of Progress in QNDE, edited by D. O. Thompson and D. E. Chimenti (Plenum, New York, 1996), Vol. 15, p. 1939.

${ }^{21}$ G. L. Petersen, C. M. Fortunko, B. B. Chick, and M. Hirao, Rev. Sci. Instrum. 65, 192 (1994).

${ }^{22}$ R. Truell, C. Elbaum, and B. Chick, Ultrasonic Methods in Solid State
Physics (Academic, New York, 1969), p. 193.

${ }^{23}$ A. B. Bhatia, J. Acoust. Soc. Am. 31, 16 (1959)

${ }^{24}$ A. Hikata, R. Truell, A. Granato, B. Chick, and K. Lücke, J. Appl. Phys. 27, 396 (1956).

${ }^{25}$ J. C. Grosskreutz and G. G. Shaw, Fatigue Crack Propagation, ASTMSTP, 415 (1967). 